\title{
Graph manifolds, left-orderability and amalgamation
}

\author{
ADAM CLAY \\ TYE LIDMAN \\ LIAM WATSON
}

\begin{abstract}
We show that every irreducible toroidal integer homology sphere graph manifold has a left-orderable fundamental group. This is established by way of a specialization of a result due to Bludov and Glass [3] for the amalgamated products that arise, and in this setting work of Boyer, Rolfsen and Wiest [6] may be applied. Our result then depends on known relations between the topology of Seifert fibred spaces and the orderability of their fundamental groups.
\end{abstract}

06F15, 20F60, 57M05

\section{Introduction}

A group $G$ is said to be left-orderable if there exists a strict total ordering $<$ of $G$ such that $g<h$ implies $f g<f h$ for all $f, g, h$ in $G$. This is equivalent to the existence of a positive cone $P \subset G$, which is a subset of elements of $G$ satisfying $P \cdot P \subset P$, and $P \sqcup\{1\} \sqcup P^{-1}=G$. To see that these notions are equivalent, given a left-ordering of a group $G$ observe that

$$
P:=\{g \in G \mid g>1\}
$$

is a positive cone; conversely if $P \subset G$ is a positive cone then

$$
g<h \Longleftrightarrow g^{-1} h \in P
$$

defines a left-ordering of $G$. We adopt the convention that $P \neq \varnothing$, so that the trivial group is not left-orderable.

Left-orderability is not preserved under many classical group operations. For example, if $G$ is a left-orderable group it is easy to see that a quotient of $G$ may or may not be left-orderable. In fact it is a relatively simple exercise to prove that a quotient $G / N$ is left-orderable if and only if $N$ is a relatively convex subgroup of $G$ (that is, $N$ is convex relative to some left-ordering on $G$ ). On the other hand, the question of left-orderability of other classical constructions is not so simple. Both HNN-extensions 
and free products with amalgamation present considerable difficulty when attempting to determine necessary and sufficient conditions for them to be left-orderable, and related problems remained open for many years (see for example [26, Problem 15.34]). However with recent work, left-orderability of free products with amalgamation and graphs of groups is now well understood (Bludov and Glass [2; 3] and Chiswell [9]).

At the same time, there has been increasing interest in left-orderable groups from a topological perspective, with particular interest in the left-orderability of the fundamental groups of 3-manifolds. Many tools in 3-manifold theory involve decomposing the manifold into simpler pieces along incompressible surfaces, resulting in a fundamental group that can be presented as a free product with amalgamation or a graph of groups. As such, these new results from the field of orderable groups should facilitate left-ordering of the fundamental groups of many 3-manifolds that contain incompressible surfaces. In particular, if a 3-manifold $M$ admits a JSJ decomposition (see Section 3, in particular Theorem 3.1) into components that are well understood from an orderability standpoint, then it should be possible to determine whether or not $\pi_{1}(M)$ is left-orderable. Our main theorem is the product of this approach.

Theorem 1.1 Let $Y$ be an irreducible, toroidal graph manifold. If $Y$ is an integer homology 3-sphere, then $\pi_{1}(Y)$ is left-orderable.

\section{A remark on conventions}

We include Seifert fibred spaces as graph manifolds; these arise when the JSJ decomposition is trivial. As such, not every toroidal graph manifold admits a non-trivial JSJ decomposition (though the converse always holds). We also include the connect sum of graph manifolds as a graph manifold. Indeed, Theorem 1.1 is a special case of Theorem 3.10 (proved in Section 3) treating the reducible case.

\section{Organization}

The plan of this paper is as follows. In Section 2, we review the necessary definitions and recent results pertaining to left-orderability of amalgamated free products. We also prove our main topological application of these results, which connects the JSJ decomposition of a 3-manifold with the manifolds obtained by Dehn filling the JSJpieces along their torus boundaries. Section 3 is devoted to the proof of Theorem 1.1. As a quick consequence, we answer a question of Dąbkowski, Przytycki and Togha [10, Problem 1(iii)]. This, together with other examples and generalizations, is provided in Section 5, while Section 4 contains a brief discussion of some of the motivation for Theorem 1.1 coming from Heegaard Floer homology. 


\section{Acknowledgements}

We thank Steve Boyer for his interest in this project, and in particular for posing the question 'does the result of \pm 4 -surgery on the figure eight have a left-orderable fundamental group?' (see Boyer, Gordon and Watson [5]), to which the answer is 'yes' (see Section 5). We also thank Stefan Friedl for comments on an early draft of this paper, as well as the referee for a thorough and thoughtful report that led to improvements throughout. The first and third authors were partially supported by NSERC postdoctoral fellowships.

\section{Requisite group theory and relevant background}

We begin with the definitions that are required to state a result of Bludov and Glass [3]. If a group $G$ is left-orderable, we denote the set of all positive cones in $G$ by $\mathrm{LO}(G)$, we think of $\operatorname{LO}(G)$ as the set of all left-orderings of $G$. The set $\operatorname{LO}(G)$ comes equipped with a natural $G$-action by conjugation, defined by $g(P)=g \mathrm{Pg}^{-1}$ for all $g \in G$ and $P \in \operatorname{LO}(G)$. In terms of left-orderings, this action can be described as follows: given a left-ordering $<$ of $G$, an element $g$ sends $<$ to the ordering $<^{g}$, which is defined according to the rule

$$
h<^{g} f \Longleftrightarrow h g<f g .
$$

Definition 2.1 A set $L \subset \mathrm{LO}(G)$ of left-orderings is normal if it is $G$-invariant.

Definition 2.2 Suppose that $G_{i}$ is a left-orderable group, $H_{i}$ is a subgroup of $G_{i}$ and let $L_{i} \subset \operatorname{LO}\left(G_{i}\right)$ denote a set of left-orderings of $G_{i}$ for $i=1$, 2. Suppose that $\phi: H_{1} \rightarrow H_{2}$ is an isomorphism. Then $\phi$ is compatible for $\left(L_{1}, L_{2}\right)$ if

$$
\left(\forall P_{1} \in L_{1}\right)\left(\exists P_{2} \in L_{2}\right) \text { such that }\left(\forall h_{1} \in H_{1}\right)\left(h_{1} \in P_{1} \Rightarrow \phi\left(h_{1}\right) \in P_{2}\right) .
$$

One of the main results of [3] is the following.

Theorem 2.3 (Bludov and Glass [3, Theorem A]) Suppose that $G_{i}$ are left-orderable groups, and $H_{i}$ is a subgroup of $G_{i}$ for $i=1,2$. Let $\phi: H_{1} \rightarrow H_{2}$ be an isomorphism. The free product with amalgamation

$$
G_{1} * G_{2}\left(H_{1} \stackrel{\phi}{\cong} H_{2}\right)
$$

is left-orderable if and only if there exist normal families $L_{i} \in \operatorname{LO}\left(G_{i}\right)(i=1,2)$ such that $\phi$ is compatible for $\left(L_{1}, L_{2}\right)$ and $\phi^{-1}$ is compatible for $\left(L_{2}, L_{1}\right)$. 
In general, using this theorem to left-order free products with amalgamation seems to be quite difficult. Unless the groups in question have a very well-understood structure, verifying the existence of normal, compatible families of left-orderings can be an intractable problem. (It is possible in some cases; see Section 5.)

However, there are several immediate corollaries of this theorem that are very useful when attempting to left-order amalgamated products, as the compatibility and normality conditions become easier to verify. The following will be most useful for our purposes.

Corollary 2.4 (Bludov and Glass [3, Corollary 5.3]) Suppose that $G_{i}$ are leftorderable groups with rank one abelian subgroups $H_{i}, i=1,2$. Let $\phi: H_{1} \rightarrow H_{2}$ be an isomorphism. Then

$$
G_{1} * G_{2}\left(H_{1} \stackrel{\phi}{\cong} H_{2}\right)
$$

is left-orderable.

Our main group-theoretic result will be an application of Theorem 2.3 to the decomposition of a 3-manifold $M$ along an incompressible torus into components $M_{1}$ and $M_{2}$ having toroidal boundaries. The key observation is that in this special case, applying Theorem 2.3 can be reduced to an application of Corollary 2.4 by using Dehn filling and the following theorem from 3-manifold topology:

Theorem 2.5 (Boyer, Rolfsen and Wiest [6, Theorem 1.1]) Suppose that $M$ is a compact, connected, $\mathbb{P}^{2}$-irreducible 3-manifold. Then $\pi_{1}(M)$ is left-orderable if and only if there exists a nontrivial homomorphism from $\pi_{1}(M)$ to a left-orderable group.

Remark This paper is concerned with integer homology spheres and as such $\mathbb{P}^{2}-$ irreducible may be replaced with irreducible by appealing to orientability.

To state and prove our key group-theoretic result, we recall the notion of Dehn filling. Suppose that $M$ is a 3-manifold with incompressible torus boundary. A slope $\alpha$ is a primitive element in the projective homology $H_{1}(\partial M ; \mathbb{Z}) /\{ \pm 1\}$ of the boundary. The result of Dehn filling $M$ along the slope $\alpha$ is the 3-manifold $M(\alpha)$ obtained by identifying the boundary of a solid torus $D^{2} \times S^{1}$ to $\partial M$ in such a way that $\partial D^{2} \times\{*\}$ is glued to $\alpha$. The following definition is natural in this setting:

Definition 2.6 Given a 3-manifold $M$ with torus boundary, a slope $\alpha$ will be called left-orderable if $\pi_{1}(M(\alpha)) \cong \pi_{1}(M) /\langle\langle\alpha\rangle\rangle$ is a left-orderable group.

It is our convention that the trivial group is not left-orderable, so any left-orderable slope $\alpha$ generates a proper normal subgroup $\langle\langle\alpha\rangle\rangle$ of $\pi_{1}(M)$ and $\pi_{1}(M(\alpha))$ is non-trivial. 
Theorem 2.7 Suppose that $M_{1}$ and $M_{2}$ are 3-manifolds with incompressible torus boundaries, and $\phi: \partial M_{1} \rightarrow \partial M_{2}$ is a homeomorphism such that $Y=M_{1} \cup_{\phi} M_{2}$ is irreducible. If there exists a left-orderable slope $\alpha$ such that $\phi_{*}(\alpha)$ is also a leftorderable slope, then $\pi_{1}(Y)$ is left-orderable (here, $\phi_{*}$ is the induced homomorphism on fundamental groups).

Proof Let $G_{i}$ denote the fundamental group $\pi_{1}\left(M_{i}\right)$ for $i=1,2$, each equipped with an inclusion $f_{i}: \mathbb{Z} \oplus \mathbb{Z} \rightarrow G_{i}$ that identifies the peripheral subgroup with $\mathbb{Z} \oplus \mathbb{Z}$, satisfying $\phi_{*} \circ f_{1}=f_{2}$. Write $q_{1}: G_{1} \rightarrow G_{1} /\langle\langle\alpha\rangle\rangle$ and $q_{2}: G_{2} \rightarrow G_{2} /\left\langle\left\langle\phi_{*}(\alpha)\right\rangle\right\rangle$ for the natural quotient maps.

Suppose that $\alpha$ and $\phi_{*}(\alpha)$ are left-orderable slopes, and consider $\langle\langle\alpha\rangle\rangle \cap \pi_{1}\left(\partial M_{1}\right)$. Since this intersection is a nontrivial subgroup of $\pi_{1}\left(\partial M_{1}\right) \cong \mathbb{Z} \oplus \mathbb{Z}$ and $\alpha$ is primitive, the intersection is isomorphic to either $\mathbb{Z} \cong\langle\alpha\rangle, \mathbb{Z} \oplus n \mathbb{Z} \subset \pi_{1}\left(\partial M_{1}\right)$, or $\mathbb{Z} \oplus \mathbb{Z} \cong \pi_{1}\left(\partial M_{1}\right)$. If $\langle\langle\alpha\rangle\rangle \cap \pi_{1}\left(\partial M_{1}\right) \cong \mathbb{Z} \oplus n \mathbb{Z}$, then the quotient $\pi_{1}(M(\alpha))$ would have torsion, so this case does not arise when $\alpha$ is a left-orderable slope. The same observation holds for the left-orderable slope $\phi_{*}(\alpha)$, so we break our proof into two cases.

First consider the case where $\langle\langle\alpha\rangle\rangle \cap \pi_{1}\left(\partial M_{1}\right)$ and $\left\langle\left\langle\phi_{*}(\alpha)\right\rangle\right\rangle \cap \pi_{1}\left(\partial M_{2}\right)$ are both infinite cyclic. Here, $\phi$ induces an isomorphism $\bar{\phi}$ between subgroups

$$
\bar{\phi}: q_{1}\left(\pi_{1}\left(\partial M_{1}\right)\right) \rightarrow q_{2}\left(\pi_{1}\left(\partial M_{2}\right)\right),
$$

satisfying $\bar{\phi} \circ q_{1} \circ f_{1}=q_{2} \circ f_{2}$. The subgroups $q_{1}\left(\pi_{1}\left(\partial M_{1}\right)\right)$ and $q_{2}\left(\pi_{1}\left(\partial M_{2}\right)\right)$ are both infinite cyclic, and by the universal property for pushouts, we have a unique homomorphism

$$
h: G_{1} *_{\phi} G_{2} \longrightarrow G_{1} /\langle\langle\alpha\rangle\rangle *_{\bar{\phi}} G_{2} /\left\langle\left\langle\phi_{*}(\alpha)\right\rangle\right\rangle
$$

resulting from the following diagram:

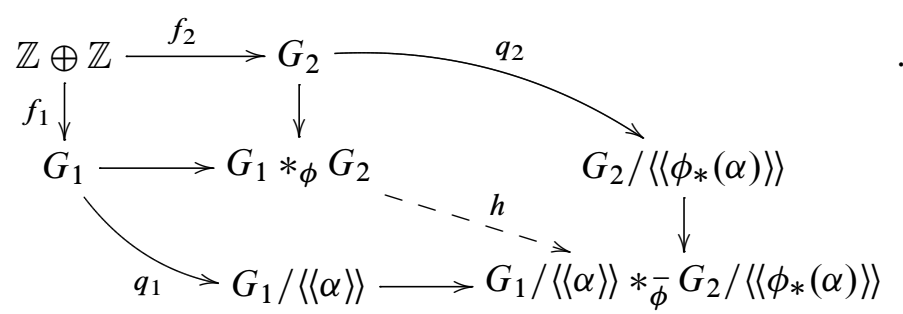

Note that $h$ is nontrivial (in fact, surjective) since the maps $q_{1}$ and $q_{2}$ are surjective.

Because the slopes $\alpha$ and $\phi_{*}(\alpha)$ are both left-orderable slopes, the group

$$
G_{1} /\langle\langle\alpha\rangle\rangle *_{\phi} G_{2} /\left\langle\left\langle\phi_{*}(\alpha)\right\rangle\right\rangle
$$


is a free product of left-orderable groups amalgamated along a cyclic subgroup. The image of the map $h$ is therefore a left-orderable group by Corollary 2.4, so that $\pi_{1}(Y) \cong G_{1} *_{\phi} G_{2}$ is left-orderable, by Theorem 2.5 .

On the other hand, suppose that either

$$
\langle\langle\alpha\rangle\rangle \cap \pi_{1}\left(\partial M_{1}\right)=\pi_{1}\left(\partial M_{1}\right), \quad \text { or } \quad\left\langle\left\langle\phi_{*}(\alpha)\right\rangle\right\rangle \cap \pi_{1}\left(\partial M_{2}\right)=\pi_{1}\left(\partial M_{2}\right),
$$

or both. Without loss of generality, suppose that $\alpha$ satisfies $\langle\langle\alpha\rangle\rangle \cap \pi_{1}\left(\partial M_{1}\right)=\pi_{1}\left(\partial M_{1}\right)$. In this setting we have an alternative construction for $h$ as follows.

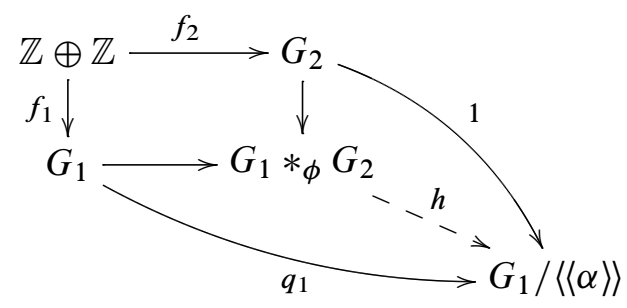

Note that this is well defined since $\langle\langle\alpha\rangle\rangle$ contains the entire peripheral subgroup $\pi_{1}\left(\partial M_{1}\right)$, and $h$ is again surjective. Now as $\alpha$ is a left-orderable slope, $G_{1} /\langle\langle\alpha\rangle\rangle$ is left-orderable and $h$ provides the required homomorphism to a left-orderable group so that $\pi_{1}(Y)$ is left-orderable by Theorem 2.5.

We record a second group theoretic proposition for later use. Recall that a splicing of knots $K_{1}$ and $K_{2}$ in homology spheres $Y_{1}$ and $Y_{2}$ is a 3-manifold $Y=M_{1} \cup_{\phi} M_{2}$ where $\phi\left(\mu_{1}\right)=\lambda_{2}$ and $\phi\left(\lambda_{1}\right)=\mu_{2}$. Here, the pair $\left\{\mu_{i}, \lambda_{i}\right\}$ is the preferred framing for each knot $K_{i}$. In particular, $\lambda_{i}$ bounds a surface in $M_{i}=Y_{i} \backslash K_{i}$ for $i=1,2$. It follows by construction that $Y$ is an integer homology sphere.

Proposition 2.8 Let $Y$ be an irreducible 3-manifold that results from splicing knots $K_{1}$ and $K_{2}$ in integer homology spheres $Y_{1}$ and $Y_{2}$ respectively. If $\pi_{1}\left(Y_{1}\right)$ is leftorderable, then $\pi_{1}(Y)$ is left-orderable.

Proof In the integer homology sphere $Y=M_{1} \cup M_{2}$, one can pinch $M_{2}$ onto a solid torus to obtain a degree one map $f: Y \rightarrow Y_{1}$. This degree one map induces a surjection $f_{*}: \pi_{1}(Y) \rightarrow \pi_{1}\left(Y_{1}\right)$, so by Theorem 2.5 the group $\pi_{1}(Y)$ is left-orderable.

\section{The proof of Theorem 1.1}

\subsection{JSJ decompositions for integer homology spheres}

Given a 3-manifold $Y$ containing an essential torus $T$ we have that $\pi_{1}(T) \cong \mathbb{Z} \oplus \mathbb{Z}$ is a subgroup of $\pi_{1}(Y)$. As a result, $\pi_{1}(Y)$ may be viewed naturally as a free product 
with amalgamated subgroup $\mathbb{Z} \oplus \mathbb{Z}$. This observation is particularly important in light of the following result of Jaco and Shalen [20], and Johannson [22].

Theorem 3.1 (Jaco and Shalen, and Johannson) Every compact, orientable, irreducible 3-manifold $Y$ with torus boundary components contains a (possibly empty) minimal collection of essential tori $\mathcal{T}$, unique up to isotopy, such that each component that results from cutting $Y$ (denoted $Y \backslash \mathcal{T}$ ) along $\mathcal{T}$ is either Seifert fibred or atoroidal.

The resulting decomposition of $Y$ along such a collection of tori is referred to as the JSJ decomposition of $Y$. Notice that there is a natural quotient from $Y$ to an underlying graph $\Gamma_{Y}$ that encodes the JSJ decomposition: the vertices are obtained by collapsing the components of $Y \backslash \mathcal{T}$ to points, and the edges correspond to collapsing $T \times I$ to the interval $I$, for each torus $T \in \mathcal{T}$. As a result we have a surjection $H_{1}(Y ; \mathbb{Z}) \rightarrow H_{1}\left(\Gamma_{Y} ; \mathbb{Z}\right)$ so that whenever $H_{1}(Y ; \mathbb{Z})=0$ the corresponding graph $\Gamma_{Y}$ is a tree. In particular, given a torus $T \in \mathcal{T}$ we have that $Y \backslash T$ is disconnected if $Y$ is an integer homology sphere; this observation will provide a key step to our induction in the proof of Theorem 1.1.

Lemma 3.2 Let $Y$ be a toroidal integer homology sphere, and suppose that $Y=$ $M_{1} \cup_{\phi} M_{2}$ where $\phi: \partial M_{1} \rightarrow \partial M_{2}$ is a homeomorphism identifying the two torus boundaries. Then $Y$ may be viewed as a splicing of knots $K_{i}$ in integer homology spheres $Y_{i}$ for $i=1,2$.

Proof Let $Y$ be a toroidal integer homology sphere, and denote the connected components of $Y \backslash T$ by $M_{1}$ and $M_{2}$. Since $Y$ is an integer homology sphere, an application of the Mayer-Vietoris theorem gives an isomorphism

$$
H_{1}\left(S^{1} \times S^{1} ; \mathbb{Z}\right) \cong H_{1}\left(M_{1} ; \mathbb{Z}\right) \oplus H_{1}\left(M_{2} ; \mathbb{Z}\right) .
$$

Applying the long exact sequence for the pair $\left(M_{i}, \partial M_{i}\right)$ we conclude that $H_{1}\left(M_{i} ; \mathbb{Z}\right) \cong \mathbb{Z}$ for $i=1,2$; let $\lambda_{i}$ denote the longitudinal slope in $\partial M_{i}$ (the unique simple closed curve that bounds in $\left.M_{i}\right)$. Note that $\left|H_{1}\left(M_{1} \cup_{f} M_{2} ; \mathbb{Z}\right)\right|=\Delta\left(f\left(\lambda_{1}\right), \lambda_{2}\right)$ for any homeomorphism $f: \partial M_{1} \rightarrow \partial M_{2}$, where $\Delta$ measures the minimal geometric intersection number between slopes.

Now denote by $\phi: \partial M_{1} \rightarrow \partial M_{2}$ the homeomorphism reconstructing $Y \cong M_{1} \cup_{\phi} M_{2}$. This homeomorphism yields a preferred meridian for each $M_{i}$ as follows. Since $\Delta\left(\phi\left(\lambda_{1}\right), \lambda_{2}\right)=1$ we define $\mu_{1}=\phi^{-1}\left(\lambda_{2}\right)$ and $\mu_{2}=\phi\left(\lambda_{1}\right)$. By construction, the pair $\left\{\mu_{i}, \lambda_{i}\right\}$ yields a basis for the peripheral subgroup of each $\pi_{1}\left(M_{i}\right)$. Now specifying $K_{i}$ in each $Y_{i}=M_{i}\left(\mu_{i}\right)$ as the core of the surgery torus produces a knot with meridian $\mu_{i}$ and longitude $\lambda_{i}$. This shows that $Y$ is a splicing of knots $K_{1}$ and $K_{2}$ in the sense that $\phi\left(\mu_{1}\right)=\lambda_{2}$ and $\phi\left(\lambda_{1}\right)=\mu_{2}$. 
We will always make this choice of preferred basis where possible. In this way, $n-$ surgery and $1 / n$-surgery on $K_{i}$ are well defined as $n \mu+\lambda$ and $\mu+n \lambda$ Dehn filling on $M_{i}$, respectively. Moreover, such a choice lets us unambiguously refer to a slope $p \mu+q \lambda$ as a reduced rational $p / q$, where $1 / 0$ denotes the trivial surgery, so that $M(p / q)$ denotes Dehn filling along the slope $p / q$. Note that it is not restrictive to assume that $q \geq 0$. By construction, $H_{1}(M(p / q)) \cong \mathbb{Z} /|p| \mathbb{Z}$.

With these observations and conventions in place, the family of manifolds considered in Theorem 1.1 are as follows:

Definition 3.3 A graph manifold is a compact, orientable, connected 3-manifold for which each prime component $Y$ has JSJ decomposition along a family of tori $\mathcal{T}$ such that the components of $Y \backslash \mathcal{T}$ are Seifert fibred.

This class of manifolds was introduced by Waldhausen [35]. Note that Seifert fibred spaces are graph manifolds; these arise precisely when $\mathcal{T}$ is empty. This definition also allows for graph manifolds obtained by connect sum of graph manifolds.

Our proof of Theorem 1.1 will be an induction on the number of tori in the collection $\mathcal{T}$, so we record the following fact.

Lemma 3.4 Let $Y$ be a graph manifold with torus boundary, and minimal collection $\mathcal{T}$ of tori decomposing $Y$ into Seifert fibred components $M_{1}, \ldots, M_{k}$. Let $\alpha$ be any slope on the boundary of $Y$. Then $Y(\alpha)$ is also a graph manifold, and $Y(\alpha)$ admits a minimal collection of tori $\mathcal{T}^{\prime}$ decomposing $Y(\alpha)$ into Seifert fibred pieces with $\left|\mathcal{T}^{\prime}\right| \leq|\mathcal{T}|$

Proof Observe that each torus in the collection $\mathcal{T}$ embeds naturally in the manifold $Y(\alpha)$. If $M_{i}$ denotes the Seifert fibred component of $Y \backslash \mathcal{T}$ that contains $\partial Y$, then $Y(\alpha) \backslash \mathcal{T}$ has components $M_{1}, \ldots, M_{i}(\alpha), \ldots, M_{k}$. By Heil [19], the manifold $M_{i}(\alpha)$ is either Seifert fibred, or is a connected sum of lens spaces and possibly copies of $S^{1} \times S^{2}$. In either case, since every manifold $M_{1}, \ldots, M_{i}(\alpha), \ldots, M_{k}$ is either Seifert fibred or a connect sum of Seifert fibred pieces, the collection $|\mathcal{T}|$ of tori embedded in $Y(\alpha)$ will cut the prime components of $Y(\alpha)$ into Seifert fibred pieces. Therefore $Y(\alpha)$ is a graph manifold. Moreover, if $\left|\mathcal{T}^{\prime}\right|$ is the collection of cutting tori from the JSJ decomposition of $Y(\alpha)$, then $\left|\mathcal{T}^{\prime}\right| \leq|\mathcal{T}|$ since $\mathcal{T}^{\prime}$ is minimal.

For repeated use in this paper, we single out a particular fact used in these arguments:

Lemma 3.5 Suppose that $M$ is Seifert fibred with torus boundary, and $H_{1}(M ; \mathbb{Z}) \cong$ $\mathbb{Z}$. Then the $0-$ filling and every $1 / n$-filling of $M$ is Seifert fibred and prime, and all but finitely many of the $n / 1-$ fillings are Seifert fibred and prime.

Proof This follows immediately from the work of Heil [19; 32]. 


\subsection{Surgeries on Seifert knots}

In this section we gather the material that will allow us to apply Theorem 2.7 and Proposition 2.8 to prove Theorem 1.1.

Theorem 3.6 (Boyer, Rolfsen and Wiest [6, Corollary 3.12]) Suppose that $Y$ is a Seifert fibred integer homology sphere. If $\pi_{1}(Y)$ is not left-orderable, then $Y$ is either $S^{3}$ or the Poincaré homology sphere $\Sigma(2,3,5)$.

This points to a strategy of proof for Theorem 1.1 that employs Theorem 2.7. Namely, we may induct in the number of Seifert pieces of a graph manifold provided some control of $S^{3}$ and $\Sigma(2,3,5)$ (in particular, Seifert fibred knots in these manifolds) may be established.

Lemma 3.7 Let $K$ be a non-trivial knot in $Y=S^{3}$ or $\Sigma(2,3,5)$ such that the complement $Y \backslash K$ is Seifert fibred. At most finitely many of the slopes $1 / n$ are not left-orderable.

Proof In light of Lemma 3.5 and Theorem 3.6, it suffices to show that $Y_{1 / n}(K)$ is not $S^{3}$ or $\Sigma(2,3,5)$ for all but finitely many $n$. If $Y=S^{3}$, then $K$ is a $(p, q)-$ torus knot. After a possible change of orientation, we may assume $p, q>0$. In this case, $S_{1 / n}^{3}(K)=\Sigma(p, q,|p q n-1|)$. This is not $S^{3}$ or $\Sigma(2,3,5)$ for $|n| \geq 2$. If $Y=\Sigma(2,3,5)$, after a choice of orientation,

$$
\begin{gathered}
Y_{1 / n}(K)=\Sigma(2,3,5,|30 n-1|), \quad \Sigma(2,3,|6 n-5|), \\
\Sigma(2,5,|10 n-3|), \quad \text { or } \quad \Sigma(3,5,|15 n-2|),
\end{gathered}
$$

depending on whether $K$ is a regular fibre, or the singular fibre of order 2, 3 or 5 respectively. Again, for $|n| \geq 2$, we never obtain $S^{3}$ or $\Sigma(2,3,5)$.

Lemma 3.8 Let $K$ be a nontrivial knot in $Y=S^{3}$ or $\Sigma(2,3,5)$ such that the complement is Seifert fibred. Then for all but finitely many positive integers or all but finitely many negative integers $n$, the slope $n / 1$ is left-orderable.

Proof For the case of $Y=S^{3}$, it is shown in Nakae [29] that for either all $\alpha \in(-1, \infty)$ or all $\alpha \in(-\infty, 1), S_{\alpha}^{3}(K)$ admits a taut foliation. When $S_{\alpha}^{3}(K)$ is Seifert fibred with base orbifold $S^{2}$, the existence of a taut foliation is equivalent to the existence of a horizontal foliation. By [6, Theorem 1.3], this is equivalent to the fundamental group being left-orderable. Therefore, $\pi_{1}\left(S_{n / 1}^{3}(K)\right)$ is left-orderable for either all $n>0$ or all $n<0$. 
Now, we consider the case that $Y=\Sigma(2,3,5)$. More generally, necessary and sufficient conditions for horizontal foliations on a Seifert manifold, and thus for left-orderability, are given explicitly in terms of the Seifert invariants in Eisenbud, Hirsch and Neumann [15], Jankins and Neumann [21], and Naimi [28]. We record two of the sufficient conditions, which will be useful for us. The first is that if a Seifert manifold $\Sigma$ with $\ell \geq 4$ singular fibres has $-(\ell-2) \leq b \leq-2$, then $\Sigma$ has left-orderable fundamental group. The second is the following. Consider a Seifert fibred space $\Sigma$ with $b=-1$ and Seifert invariants $b_{1} / a_{1}<\cdots<b_{\ell} / a_{\ell}$, where $0<b_{i}<a_{i}$. If there exist relatively prime integers $0<k<m$ and a permutation of $\sigma$ of the indices such that $b_{\sigma(1)} / a_{\sigma(1)}<k / m$, $b_{\sigma(2)} / a_{\sigma(2)}<(m-k) / m$ and $b_{\sigma(i)} / a_{\sigma(i)}<1 / m$ for all other $i$, then $\Sigma$ has leftorderable fundamental group.

First, consider the case of $n / 1$-surgery on a regular fibre in $\Sigma(2,3,5)$. For all but finitely many $n$, this will be a Seifert manifold with 4 singular fibres and $b=-2$. Therefore, $\pi_{1}$ will be left-orderable. We now consider the case that $K$ is a singular fibre. In this case, the result of $n / 1$-surgery will generically be a Seifert manifold with three-singular fibres and $b=-1$. Up to orientation, the Seifert invariants for $n / 1-$ surgery on the singular fibres of order 2,3 and 5 are given by $\{1 / 3,3 / 5,1 /(15-n)\}$, $\{1 / 2,2 / 5,1 /(10-n)\}$ and $\{1 / 2,1 / 3,1 /(6-n)\}$, respectively. Each of these manifolds satisfies the second sufficient condition discussed above for all $n<0$.

We also record the following similar lemma for use in the proof of Theorem 1.1, which is essentially a compilation of known surgery results.

Lemma 3.9 Suppose that $K \subset Y$ is a knot in an integer homology sphere graph manifold $Y, \pi_{1}(Y)$ is not left-orderable, and set $M=Y \backslash K$. Then $M(1 / n)$ is irreducible and $\pi_{1}(M(1 / n))$ is infinite for all but finitely many $n$.

Proof First suppose that $M$ is Seifert fibred. Since $Y$ is not left-orderable, $M$ is the complement of a knot in $S^{3}$ or in $\Sigma(2,3,5)$, as already observed, Lemma 3.5 shows that $M(1 / n)$ is irreducible for all but finitely many slopes. The result in this case then follows from Lemma 3.7 .

If $M$ is not Seifert fibred (i.e., $M$ contains a JSJ torus) then at most three of the manifolds $M(1 / n)$ are reducible, by Gordon and Luecke [17]. To show that only finitely many surgeries on a manifold $M$ containing a JSJ torus can yield a finite fundamental group, we consider two cases. First, if $M$ does not decompose along incompressible tori into one or two cable spaces and a Seifert fibred space over the 2-disk with two exceptional fibres, then at most three slopes yield a finite fundamental group, by Theorem B of Boyer and Zhang [7]. In the case that $M$ does admit such a 
decomposition, then at most six slopes yield a finite fundamental group, by Theorems 6 and 8 of Bleiler and Hodgson [1].

\subsection{A generalization and proof of Theorem 1.1}

We now have the material in place to prove Theorem 1.1, which is an immediate consequence of the following:

Theorem 3.10 Let $Y$ be a graph manifold other than $S^{3}$, none of whose prime components is a Poincaré homology sphere. If $Y$ is an integer homology sphere, then $\pi_{1}(Y)$ is left-orderable.

Proof First, observe that if $Y=Y_{1} \# \cdots \# Y_{m}$, then $\pi_{1}(Y)$ is a free product of the groups $\pi_{1}\left(Y_{i}\right)$. It is well known that if $G$ is a free product of the groups $G_{i}$, then $G$ is left-orderable if and only if each nontrivial $G_{i}$ is left-orderable (Vinogradov [34]). Therefore, $\pi_{1}(Y)$ will be left-orderable if and only if each nontrivial $\pi_{1}\left(Y_{i}\right)$ is left-orderable. Hence, for the remainder of the proof we may assume that $Y$ is prime.

We now proceed by induction on the number of tori in the family $\mathcal{T}$ provided by the JSJ decomposition of $Y$, as given by Theorem 3.1. First, if $\mathcal{T}=\varnothing$, then $Y$ is Seifert fibred. Since $Y$ is not $S^{3}$ or $\Sigma(2,3,5)$ by assumption, Theorem 3.6 ensures that $\pi_{1}(Y)$ is left-orderable.

The first case of interest is when $|\mathcal{T}|=1$. In particular, $Y$ is a splicing of $Y_{1}$ and $Y_{2}$ along knots $K_{1}$ and $K_{2}$, as in Lemma 3.2, such that each knot complement $M_{i}=Y_{i} \backslash K_{i}$ is a boundary-irreducible Seifert fibred space. We denote the gluing map by $\phi$, so that $Y=M_{1} \cup_{\phi} M_{2}$. Observe that $Y_{1}$ and $Y_{2}$ must be Seifert fibred spaces as well by applying Lemma 3.5 to the slope $1 / 0$.

First suppose that one of the $Y_{i}$ has a left-orderable fundamental group. Since $Y$ is irreducible, by Proposition $2.8, \pi_{1}(Y)$ is left-orderable.

Therefore, we can assume $\pi_{1}\left(Y_{1}\right)$ and $\pi_{1}\left(Y_{2}\right)$ are both non-left-orderable groups, so that each $Y_{i}$ is one of $S^{3}$ or $\Sigma(2,3,5)$. By Lemma 3.8, either infinitely many positive or negative integer slopes on $\partial M_{1}$ are left-orderable; by Lemma 3.7 only finitely many slopes on $\partial M_{2}$ of the form $1 / n$ are not left-orderable. Recall that since the homeomorphism $\phi$ specifies a splicing of knots, relative to our chosen bases of the peripheral subgroups $\phi$ sends the slope $p / q$ on $\partial M_{1}$ to the slope $q / p$ on $\partial M_{2}$. Therefore, we can find a pair of left-orderable slopes of the form $n / 1$ on $M_{1}$ and $1 / n$ on $M_{2}$ that are identified by $\phi$. Theorem 2.7 completes the proof in this setting, and provides a base case for induction. 
We now provide the induction step. Assume that every irreducible integer homology sphere graph manifold whose JSJ decomposition has fewer than $p$ tori (other than $S^{3}$ or $\Sigma(2,3,5))$ has left-orderable fundamental group. Suppose that $Y$ has tori $T_{1}, \ldots, T_{p}$ in the JSJ decomposition, where $p \geq 2$. Since $\Gamma_{Y}$ is a tree, $T_{p}$ is a separating torus and $Y \backslash T_{p}$ consists of the two manifolds $M_{i}=Y_{i} \backslash K_{i}$, each containing $p-1$ or fewer tori in its JSJ decomposition. Without loss of generality, we can choose $T_{p}$ such that $M_{2}$ is Seifert fibred. By Lemma 3.4, the number of tori in the JSJ decomposition of $Y_{1}=M_{1}\left(\mu_{1}\right)$ is less than or equal to $p-1$.

There are two cases to consider. First suppose that $\pi_{1}\left(Y_{2}\right)$ is left-orderable. Proposition 2.8 applies and we conclude that $\pi_{1}(Y)$ is left-orderable as well. On the other hand if $\pi_{1}\left(Y_{2}\right)$ is not left-orderable then $Y_{2}$ is either $S^{3}$ or $\Sigma(2,3,5)$, hence all but finitely many slopes of the form $n / 1$ in $\partial M_{2}$ are not left-orderable (for an appropriate choice of the sign of $n$ ) by Lemma 3.8. On the other hand, by Lemma 3.9 there are only finitely many slopes $1 / n$ in $\partial M_{1}$ for which $M_{1}(1 / n)$ is reducible or $\pi_{1}\left(M_{1}(1 / n)\right)$ is finite. Thus, by the induction hypothesis there are only finitely many slopes $1 / n$ in $\partial M_{1}$ that are not left-orderable. It follows that for $n$ sufficiently large and for an appropriate choice of sign, there exist left-orderable slopes $1 / n$ and $n / 1$ in $\partial M_{1}$ and $\partial M_{2}$, respectively, and an application of Theorem 2.7 concludes the proof.

\section{Relationship with Heegaard Floer homology}

We briefly explain some of the motivation for Theorem 1.1. This stems from the following two conjectures pertaining to $\mathrm{L}$-spaces. Recall that an $\mathrm{L}-$-space is a rational homology sphere with $\left|H_{1}(Y ; \mathbb{Z})\right|$ equal to the rank of $\widehat{H F}(Y)$, the "hat" version Heegaard Floer homology of $Y$.

Conjecture 4.1 (Boyer, Gordon and Watson [5]) If $Y$ is a prime rational homology sphere, then $Y$ is an $L$-space if and only if $\pi_{1}(Y)$ is not left-orderable.

Conjecture 4.2 (Ozsváth and Szabó) If $Y$ is a prime L-space integer homology sphere, then $Y=S^{3}$ or $\Sigma(2,3,5)$.

Note that Conjecture 4.1 holds when $Y$ is a Seifert manifold [5, Theorem 1]. There also exist a number of independent proofs of Conjecture 4.2 for Seifert fibred rational homology spheres, providing a Heegaard Floer homology analogue of Theorem 3.6. In fact, one way to see this is by combining Theorem 3.6 with the affirmation of Conjecture 4.1 for Seifert manifolds. 
A natural next step is to consider toroidal manifolds, a study first undertaken by Eftekhary [14]. Boyer and Boileau show in [4] that every integer homology sphere graph manifold other than $S^{3}$ or $\Sigma(2,3,5)$ admits a smooth, co-orientable taut foliation, and so is not an L-space (Ozsváth and Szabó [30, Theorem 1.4]). More generally, Hedden and Levine use bordered Floer homology to prove that if $Y$ is a toroidal integer homology sphere then $Y$ is not an L-space, under the hypothesis that $Y$ is obtained by splicing knots in integer homology sphere L-spaces [18, Theorem 1]. The general question of showing that a toroidal integer homology sphere is an L-space (i.e., removing the splicing hypothesis from [18, Theorem 1]) remains an open problem [18, Conjecture 2].

With these results in mind, it is natural to ask what can be said about the left-orderability of the fundamental groups of integer homology spheres, which was our motivation for Theorem 1.1. While the proof presented in the previous section does not rely on Heegaard Floer homology, in the course of our work we found an alternative HeegaardFloer theoretic proof of Theorem 1.1, which was suggestive of a possible approach to proving [18, Conjecture 2]. Consider the following analogue of Theorem 2.7:

Conjecture 4.3 Let $M_{1}$ and $M_{2}$ be 3-manifolds with incompressible torus boundaries, and $\phi: \partial M_{1} \rightarrow \partial M_{2}$ a homeomorphism. If there exists a slope $\alpha$ such that $M_{1}(\alpha)$ and $M_{2}\left(\phi_{*}(\alpha)\right)$ are not $L-$ spaces, then $Y=M_{1} \cup_{\phi} M_{2}$ is not an $L$-space.

Suppose $Y$ is a toroidal integer homology sphere. We again write $Y$ as a splice of $\left(Y_{1}, K_{1}\right)$ and $\left(Y_{2}, K_{2}\right)$. Analogous to Lemma 3.7, it can be shown that $1 / n$-surgery on $K_{1}$ is not an L-space for all but finitely many $n$ (regardless of geometric structure on $Y_{1}$ or $K_{1}$ ). Similar to Lemma 3.8, either for $n \gg 0$ or $n \ll 0, n / 1$-surgery on $K_{2}$ cannot be an L-space. We are now able to identify a pair of non-L-space slopes under the gluing map, and Conjecture 4.3 would show that $Y$ is not an $\mathrm{L}-$ space. It is worth mentioning that the work of Hedden and Levine [18] suggests that bordered Heegaard Floer homology is an essential object in this setting.

\section{Examples, consequences and generalizations}

\subsection{On a question of Dąbkowski, Przytycki and Togha}

As an immediate consequence of Theorem 1.1, we provide a negative answer to [10, Problem 1(iii)].

Proposition 5.1 There exists a hyperbolic knot $K$ in $S^{3}$ with $n$-fold cyclic-branched cover $\Sigma_{n}(K)$ that has left-orderable fundamental group and finite $H_{1}\left(\Sigma_{n}(K) ; \mathbb{Z}\right.$ ) (for infinitely many positive integers $n$ ). 
Proof Let $K$ be the Conway knot (the knot $11 n 34$ in the standard knot tables; its mutant, the Kinoshita-Terasaka knot, is the knot $11 n 42)$. As $\operatorname{det}(K)=1$, the two-fold cyclic branched cover of $K$ has trivial first-homology. Montesinos shows that $\Sigma_{2}(K)$ can be obtained by surgery on a connect-sum of two trefoil knots [27]. This surgery results in an irreducible toroidal integer homology sphere $Y \cong M_{1} \cup M_{2}$; each $M_{i}$ is homeomorphic to a trefoil exterior. Since the trefoil is a torus knot, its complement is Seifert fibred. Therefore, $\Sigma_{2}(K)$ is an irreducible toroidal graph manifold and $\pi_{1}\left(\Sigma_{2}(K)\right)$ is left-orderable by Theorem 1.1. We remark that the JSJ decomposition of $\Sigma_{2}(K)$ may be deduced by inspection of the branch set (see [27], for example).

Now we can easily extend this example to obtain infinitely many $n$ such that $\Sigma_{n}(K)$ is left-orderable and $H_{1}\left(\Sigma_{n}(K) ; \mathbb{Z}\right)$ finite. A theorem of Fox states that $H_{1}\left(\Sigma_{n}(K) ; \mathbb{Z}\right)$ will be infinite if and only if the Alexander polynomial for $K$ has zeros at $n^{\text {th }}$ roots of unity (Weber [36]). Since the Conway knot has Alexander polynomial 1, $H_{1}\left(\Sigma_{n}(K) ; \mathbb{Z}\right)$ is always finite.

If $2 \mid n$ then $\Sigma_{n}(K)$ is an $m$-fold cover of $\Sigma_{2}(K)$ where $m=n / 2$. To see this, recall that $\pi_{1}\left(\Sigma_{n}(K)\right)$ may be viewed as the group $K^{n}=\operatorname{ker}\left(\pi_{1}(K) \rightarrow \mathbb{Z} / n \mathbb{Z}\right) /\left\langle\left\langle\mu^{n}\right\rangle\right\rangle$ where $\mu$ is the meridian of $K$. There is an obvious inclusion of $\operatorname{ker}\left(\pi_{1}(K) \rightarrow \mathbb{Z} / n \mathbb{Z}\right) \subset$ $\operatorname{ker}\left(\pi_{1}(K) \rightarrow \mathbb{Z} / 2 \mathbb{Z}\right)$ when $2 \mid n$, and this descends to give a homomorphism $K^{n} \rightarrow K^{2}$. In particular, there is a non-trivial homomorphism $\pi_{1}\left(\Sigma_{n}(K)\right) \rightarrow \pi_{1}\left(\Sigma_{2}(K)\right)$ when $n$ is even.

Finally, since the Conway knot is prime, $\Sigma_{n}(K)$ is irreducible (Plotnick [31]). The observation that $\pi_{1}\left(\Sigma_{2}(K)\right)$ is left-orderable, together with the homomorphism

$$
\pi_{1}\left(\Sigma_{n}(K)\right) \rightarrow \pi_{1}\left(\Sigma_{2}(K)\right),
$$

implies that $\pi_{1}\left(\Sigma_{n}(K)\right)$ is left-orderable (applying Theorem 2.5) whenever $n$ is even.

We remark that, since the Conway knot is hyperbolic, the manifold $\Sigma_{n}(K)$ is hyperbolic for all sufficiently large $n$ by work of Thurston [33].

Remark One can also find infinitely many examples as in Proposition 5.1 in another way. Let $K_{m}$ be the $(-2 m-1,4 m+1,4 m+3)$-pretzel knot for $m \geq 1$. Note that $\Delta_{K_{m}}=1$. The branched double-cover of $K_{m}$ will be the Brieskorn sphere $\Sigma(2 m+1,4 m+1,4 m+3)$. Since $m \geq 1$, this manifold will not be $S^{3}$ or $\Sigma(2,3,5)$, and therefore has left-orderable fundamental group. That these knots are hyperbolic follows from Kim and Lee [23, Theorem 2.2 and Theorem 2.4]. Again, using $\Sigma_{2 n}\left(K_{m}\right)$ as in the proof of Proposition 5.1 provides examples where the manifolds are hyperbolic rational homology spheres as well. 


\subsection{Hyperbolic pieces}

It is natural to ask how Theorem 1.1 might generalize to toroidal 3-manifolds with hyperbolic pieces.

Theorem 5.2 Let $Y$ be a toroidal integer homology sphere graph manifold with nontrivial JSJ decomposition, and fix a knot $K$ in some Seifert piece of $Y$. Consider $M^{\prime}=Y^{\prime} \backslash K^{\prime}$, the exterior of some knot $K^{\prime}$ in an integer homology sphere $Y^{\prime}$ for which the longitudinal filling $M^{\prime}\left(\lambda^{\prime}\right)$ is prime. Then the fundamental group of the manifold resulting from the splicing of $K$ and $K^{\prime}$ is left-orderable.

Proof Let $\mu$ denote the meridian of $K$, and note that $\mu$ is a left-orderable slope by Theorem 1.1. Now the manifold resulting from splicing $K$ and $K^{\prime}$ has left-orderable fundamental group by Proposition 2.8.

Notice that $M^{\prime}$ in Theorem 5.2 may be hyperbolic, and that it is straightforward to iterate this construction with any number of hyperbolic pieces. This yields a large class of toroidal integer homology spheres with left-orderable fundamental groups, in the spirit of Theorem 1.1, containing hyperbolic pieces.

We remark that in general there seems to be a strong interaction with co-orientable taut foliations when considering the question of left-orderable fundamental groups among integer homology spheres. For example, Li and Roberts show that for every knot in $S^{3}$ there is a neighbourhood of 0 such that every slope in this neighbourhood yields a manifold admitting a co-orientable taut foliation [24]. Calegari and Dunfield have proved that an atoroidal integer homology sphere admitting a co-orientable taut foliation has left-orderable fundamental group [8, Corollary 7.6]. Combining these results yields a large class of hyperbolic integer homology spheres with left-orderable fundamental groups by considering $1 / n$-surgery on a hyperbolic knot in $S^{3}$ for all but finitely many $n$. This provides a potentially useful tool for constructing further infinite families along the lines of Theorem 5.2.

\subsection{Toroidal graph manifolds in general}

While Theorem 2.7 is sufficient to deal with integer homology sphere graph manifolds, it is not sufficient to deal with all rational homology sphere graph manifolds. However, in this setting it is sometimes possible to apply Theorem 2.3 directly in order to leftorder the fundamental group of a given graph manifold. The example that follows illustrates this fact. 
Recall that the braid group $B_{n}$ is the group generated by $\sigma_{1}, \ldots, \sigma_{n-1}$, subject to the relations $\sigma_{i} \sigma_{j} \sigma_{i}=\sigma_{j} \sigma_{i} \sigma_{j}$ if $|i-j|=1$, and $\sigma_{i} \sigma_{j}=\sigma_{j} \sigma_{i}$ if $|i-j|>1$. The braid group $B_{3}$ is isomorphic to the fundamental group of the complement of the trefoil; viewed as such the meridian is $\mu=\sigma_{2}$ and the longitude is $\lambda=\Delta^{2} \sigma_{2}^{-6}$, where

$$
\Delta=\sigma_{1} \sigma_{2} \sigma_{1} \text {. }
$$

Relative to this basis of the peripheral subgroup, the fibre slope of the Seifert structure is $6 \mu+\lambda=\Delta^{2}$.

Next, we consider the twisted $I$-bundle over the Klein bottle, which has fundamental group

$$
\left\langle x, y \mid x y x^{-1}=y^{-1}\right\rangle,
$$

with peripheral subgroup $\left\langle y, x^{2}\right\rangle$. There are two possible Seifert structures on this manifold. The first Seifert structure has a Möbius band as base orbifold, with no cone points, and the regular fibre in this case is represented by $y$. The second Seifert structure has a disk as base orbifold, with two cone points of multiplicity 2 . In this case the regular fibre is given by $x^{2}$.

We now define our graph manifold of interest. Let $M_{1}$ denote the complement of the trefoil, and $M_{2}$ the twisted $I$-bundle over the Klein bottle, with fundamental groups as described above. Define

$$
M:=M_{1} \cup_{\phi} M_{2}
$$

where $\phi$ is the gluing map of their boundaries defined on the peripheral subgroups by the formula

$$
\phi\left(\sigma_{2}\right)=y^{-1}, \quad \phi\left(\Delta^{2}\right)=y^{-1} x^{2} .
$$

By applying the Seifert-Van Kampen theorem, we see that $\pi_{1}(M)=\left\langle\sigma_{1}, \sigma_{2}, x, y \mid \sigma_{1} \sigma_{2} \sigma_{1}=\sigma_{2} \sigma_{1} \sigma_{2}, x y x^{-1}=y^{-1}, \sigma_{2}=y^{-1}, \Delta^{2}=y^{-1} x^{2}\right\rangle$. It is not hard to check that $\pi_{1}(M)$ abelianizes to give $\mathbb{Z} / 4 \mathbb{Z}$, so $M$ is not an integer homology sphere (In fact, $M$ arises from +4 -surgery on the figure eight knot and came to the attention of the authors as a result of left-ordering manifolds arising from Dehn surgery on the figure eight knot; see [5]). Moreover, since the map $\phi$ does not send the unique Seifert fibre slope on $\partial M_{1}$ to either of the Seifert fibre slopes on $\partial M_{2}$, the manifold $M$ is not Seifert fibred, so is a toroidal graph manifold.

Proposition 5.3 The fundamental group of $M$ cannot be left-ordered by applying Theorem 2.7. 
Proof In order to apply Theorem 2.7, we must find a left-orderable slope $s$ that is mapped to a left-orderable slope $\phi(s)$ by $\phi$. The twisted $I$-bundle over the Klein bottle has only one left-orderable slope, corresponding to the group element $y$ in the presentation above. In particular, filling along any other slope gives a manifold with elliptic geometry and finite fundamental group, or a fundamental group that is a free product of finite groups in the case of filling along the slope $x^{2}$. Therefore, if we are to apply Theorem 2.7 to the graph manifold $M$, we must take $\phi(s)=y$ to be our left-orderable slope on $\partial M_{2}$. Correspondingly, we must have $s=\phi^{-1}(y)=\sigma_{2}=\mu$. However, $\mu=\sigma_{2}$ is not a left-orderable slope since $B_{3} /\left\langle\left\langle\sigma_{2}\right\rangle\right\rangle$ is trivial. We conclude that Theorem 2.7 cannot be applied in this case.

With a little work, we can show that $\pi_{1}(M)$ is left-orderable by applying Theorem 2.3. The success of this approach relies heavily on the fact that the left-orderings of $B_{3}$ are very well studied. First we need some definitions and lemmas.

Recall that a word $w$ in the generators $\sigma_{1}, \sigma_{2}$ is 1 -positive if $\sigma_{1}$ occurs with only positive exponents and $1-$ negative if $\sigma_{1}$ has only negative exponents. We define a leftordering of $B_{3}$, called the Dubrovina-Dubrovin ordering, according to the following rule: $\beta \in B_{3}$ is positive if $\beta$ admits a 1-positive representative word in the generators $\sigma_{1}, \sigma_{2}$, or if $\beta=\sigma_{2}^{k}$ for some $k<0$. Denote this left-ordering of $B_{3}$ by $<_{D D}$. For background on this left-ordering of $B_{3}$ and the related Dehornoy ordering, see Dehornoy [11], Dehornoy, Dynnikov, Rolfsen and Wiest [12], and Dubrovina and Dubrovin [13]. An important property for our purposes is:

Lemma 5.4 (Special case of Property S [12]) Let $\beta$ be any braid; suppose that $k>0$ and $\beta^{-1} \sigma_{2}^{k} \beta$ is not a power of $\sigma_{2}$. The braid $\beta^{-1} \sigma_{2}^{k} \beta$ is 1 -positive if and only if $k>0$.

Lemma 5.5 (Malyutin [25]; see [12, Lemma 3.4]) Given $\alpha, \beta \in B_{3}$, the following hold for every left-ordering $<$ of $B_{3}$ and every pair of integers $k, l$ :

(1) $\alpha<\Delta^{2 k}$ and $\beta<\Delta^{2 l}$ implies $\alpha \beta<\Delta^{2(k+l)}$,

(2) $\alpha>\Delta^{2 k}$ and $\beta>\Delta^{2 l}$ implies $\alpha \beta>\Delta^{2(k+l)}$,

(3) $\alpha<\Delta^{2 k}$ implies $\Delta^{-2 k}<\alpha^{-1}$.

We use these facts without explicit reference in the proofs that follow.

Lemma 5.6 Let $\beta \in B_{3}$ be any braid, and $k$ any integer. Then

$$
\Delta^{-2}<_{D D} \beta^{-1} \sigma_{2}^{k} \beta<_{D D} \Delta^{2} \text {. }
$$


Proof For contradiction, suppose that $\Delta^{2}{ }_{{ }_{D D}} \beta^{-1} \sigma_{2}^{k} \beta$. Since $\Delta$ is cofinal in every left-ordering of $B_{3}$ [12], we may choose $m$ so that $\Delta^{2 m}{ }_{D D D} \beta{ }_{D D} \Delta^{2 m+2}$, hence $\Delta^{-2 m-2}<D D \beta^{-1}<D D \Delta^{-2 m}$.

By squaring elements in the first inequality, we get $\Delta^{4}{ }_{{ }_{D D D}} \beta^{-1} \sigma_{2}^{2 k} \beta$, since $\Delta^{2}$ is central. Combining this with the lower bounds for $\beta$ and $\beta^{-1}$, we get:

$$
\Delta^{2}=\Delta^{2 m} \cdot \Delta^{4} \cdot \Delta^{-2 m-2}{ }_{D D D} \beta \cdot \beta^{-1} \sigma_{2}^{2 k} \beta \cdot \beta^{-1}=\sigma_{2}^{2 k} .
$$

We conclude $\Delta^{2}{ }_{D D D} \sigma_{2}^{2 k}$, or $\sigma_{2}^{-2 k} \Delta^{2}<_{D D} 1$, which is not possible since $\sigma_{2}^{-2 k} \Delta^{2}$ is 1 -positive. This contradiction completes the proof. The inequality $\Delta^{-2}{ }{ }_{D D} \beta^{-1} \sigma_{2}^{k} \beta$ is proved in a similar fashion.

Lemma 5.7 Every conjugate of the Dubrovina-Dubrovin ordering of $B_{3}$ restricts to one of the following two orderings on the subgroup $\left\langle\sigma_{2}, \Delta^{2}\right\rangle$ :

(1) $\sigma_{2}^{k} \Delta^{2 l}$ is positive if $l>0$, or $l=0$ and $k>0$, or

(2) $\sigma_{2}^{k} \Delta^{2 l}$ is positive if $l>0$, or $l=0$ and $k<0$.

Proof Let $\beta \in B_{3}$ be any braid. If $\sigma_{2}^{k} \Delta^{2 l}$ is positive in the conjugate ordering $<_{D D}^{\beta}$, then by definition we have

$$
\beta<{ }_{D D} \sigma_{2}^{k} \Delta^{2 l} \beta,
$$

which we can rearrange to give $\beta^{-1} \sigma_{2}^{-k} \beta{ }_{D D D} \Delta^{2 l}$. By Lemma 5.6, either $l>0$, or $l=0$ and we have $\beta^{-1} \sigma_{2}^{-k} \beta<_{D D} 1$.

We now consider two cases. First, if $\beta$ commutes with $\sigma_{2}$ (and hence all its powers by Fenn, Rolfsen and Zhu [16]), then $\beta^{-1} \sigma_{2}^{-k} \beta<_{D D} 1$ becomes $\sigma_{2}^{-k}<_{D D} 1$, which happens if and only if $k<0$.

Second, if $\beta$ does not commute with $\sigma_{2}$, then $\beta^{-1} \sigma_{2}^{-k} \beta$ is not a power of $\sigma_{2}$. By definition of the DD-ordering, $\beta^{-1} \sigma_{2}^{-k} \beta{ }_{D D} 1$ implies that $\beta^{-1} \sigma_{2}^{-k} \beta$ is 1 -negative. By Property S, this happens if and only if $k>0$.

Proposition 5.8 The fundamental group $\pi_{1}(M)=\left\langle\sigma_{1}, \sigma_{2}, x, y \mid \sigma_{1} \sigma_{2} \sigma_{1}=\sigma_{2} \sigma_{1} \sigma_{2}, x y x^{-1}=y^{-1}, \sigma_{2}=y^{-1}, \Delta^{2}=y^{-1} x^{2}\right\rangle$ is left-orderable.

Proof Recall that $M$ is the union of two Seifert fibred pieces $M_{1}$ and $M_{2}$ with $\pi_{1}\left(M_{1}\right)=B_{3}$ and $\pi_{1}\left(M_{2}\right)=\left\langle x, y \mid x y x^{-1}=y^{-1}\right\rangle$, with the gluing map

$$
\phi:\left\langle\sigma_{2}, \Delta^{2}\right\rangle \rightarrow\left\langle y, x^{2}\right\rangle
$$


given by the formula

$$
\phi\left(\sigma_{2}\right)=y^{-1}, \phi\left(\Delta^{2}\right)=y^{-1} x^{2} .
$$

In order to use Theorem 2.7 and show that $\pi_{1}(M)$ is left-orderable, we must define normal families $L_{1} \subset \mathrm{LO}\left(\pi_{1}\left(M_{1}\right)\right)$ and $L_{2} \subset \operatorname{LO}\left(\pi_{1}\left(M_{2}\right)\right)$ that are compatible with the map $\phi$.

The first normal family, $L_{1}$, will be all conjugates of the Dubrovina-Dubrovin ordering of $B_{3}$, as defined above. For the normal family $L_{2}$, consider the short exact sequence

$$
1 \rightarrow\langle\langle y\rangle\rangle \rightarrow \pi_{1}\left(M_{2}\right) \stackrel{q}{\rightarrow}\langle x\rangle \rightarrow 1 .
$$

We can use this short exact sequence to define two left-orderings $<_{1}$ and $<_{2}$ of $\pi_{1}\left(M_{2}\right)$ that are conjugate to each other by the action of $x$. Given $g \in \pi_{1}\left(M_{2}\right)$, declare $1<_{1} g$ if $q(g)=x^{l}, l>0$, or if $q(g)=1$ and $g=y^{k}$ with $k>0$. Similarly, define $1<_{2} g$ if $q(g)=x^{l}, l>0$, or if $q(g)=1$ and $g=y^{k}$ with $k<0$, so that the sign of $y$ is opposite in $<_{2}$. It is not hard to check that $L_{2}=\left\{<_{1},<_{2}\right\}$ is a normal family of $\operatorname{LO}\left(\pi_{1}\left(M_{2}\right)\right)$.

Now we check that the normal families $L_{1}$ and $L_{2}$ are compatible with the map $\phi$. To this end, choose a left-ordering $<$ from the family $L_{1}$. There are two cases to consider.

Case 1 The ordering $<$ is conjugate to $<_{D D}$ by a braid $\beta$ that does not commute with $\sigma_{2}$. In this case, we use the ordering $<_{2}$ in $L_{2}$ to demonstrate compatibility. Choose $h=\sigma_{2}^{k} \Delta^{2 l} \in\left\langle\sigma_{1} \Delta^{2}\right\rangle$ satisfying $1<h$, then by Lemma 5.7 either $l>0$ or $l=0$ and $k>0$. Then

$$
\phi(h)=y^{-k}\left(y^{-1} x^{2}\right)^{l},
$$

and we wish to check that $1<_{2} y^{-k}\left(y^{-1} x^{2}\right)^{l}$. Observe that $q\left(y^{-k}\left(y^{-1} x^{2}\right)^{l}\right)=x^{2 l}$, so $1<_{2} y^{-k}\left(y^{-1} x^{2}\right)^{l}$ if $l>0$. If $l=0$, then $y^{-k}\left(y^{-1} x^{2}\right)^{l}=y^{-k}$, and $1<_{2} y^{-k}$ because $k>0$.

Case 2 The ordering $<$ is conjugate to ${ }_{D D D}$ by a braid $\beta$ that commutes with $\sigma_{2}$. In this case we use $<_{1}$ in $L_{1}$ to demonstrate compatibility with $\phi$. The calculation is nearly identical to Case 1 (with the signs of $k$ reversed), and is left to the reader.

\section{References}

[1] S A Bleiler, C D Hodgson, Spherical space forms and Dehn filling, Topology 35 (1996) 809-833 MR1396779

[2] V V Bludov, A M W Glass, Conjugacy in lattice-ordered groups and right ordered groups, J. Group Theory 11 (2008) 623-633 MR2446144 
[3] V V Bludov, A M W Glass, Word problems, embeddings, and free products of rightordered groups with amalgamated subgroup, Proc. Lond. Math. Soc. 99 (2009) 585-608 MR2551464

[4] S Boyer, M Boileau, Graph manifolds $\mathbb{Z}$-homology 3-spheres and taut foliations arXiv: 1303.5264

[5] S Boyer, C M Gordon, L Watson, On L-spaces and left-orderable fundamental groups, Math. Annalen (2012) 1-33

[6] S Boyer, D Rolfsen, B Wiest, Orderable 3-manifold groups, Ann. Inst. Fourier (Grenoble) 55 (2005) 243-288 MR2141698

[7] S Boyer, X Zhang, Exceptional surgery on knots, Bull. Amer. Math. Soc. 31 (1994) 197-203 MR1260518

[8] D Calegari, N M Dunfield, Laminations and groups of homeomorphisms of the circle, Invent. Math. 152 (2003) 149-204 MR1965363

[9] I M Chiswell, Right orderability and graphs of groups, J. Group Theory 14 (2011) 589-601 MR2818951

[10] M K Da̧bkowski, J H Przytycki, A A Togha, Non-left-orderable 3-manifold groups, Canad. Math. Bull. 48 (2005) 32-40 MR2118761

[11] P Dehornoy, Braid groups and left distributive operations, Trans. Amer. Math. Soc. 345 (1994) 115-150 MR1214782

[12] P Dehornoy, I Dynnikov, D Rolfsen, B Wiest, Ordering braids, Mathematical Surveys and Monographs 148, American Mathematical Society (2008) MR2463428

[13] T V Dubrovina, N I Dubrovin, On braid groups, Mat. Sb. 192 (2001) 53-64 MR1859702 In Russian; translated in Sb. Math. 192:5-6 (2001) 693-703

[14] E Eftekhary, Floer homology and existence of incompressible tori in homology spheres arXiv:0807.2326

[15] D Eisenbud, U Hirsch, W D Neumann, Transverse foliations of Seifert bundles and self-homeomorphism of the circle, Comment. Math. Helv. 56 (1981) 638-660 MR656217

[16] R Fenn, D Rolfsen, J Zhu, Centralisers in the braid group and singular braid monoid, Enseign. Math. 42 (1996) 75-96 MR1395042

[17] C M Gordon, J Luecke, Reducible manifolds and Dehn surgery, Topology 35 (1996) 385-409 MR1380506

[18] M Hedden, A S Levine, Splicing knot complements and bordered Floer homology arXiv: 1210.7055

[19] W Heil, Elementary surgery on Seifert fiber spaces, Yokohama Math. J. 22 (1974) 135-139 MR0375320 
[20] W Jaco, PB Shalen, Seifert fibered spaces in irreducible, sufficiently-large 3manifolds, Bull. Amer. Math. Soc. 82 (1976) 765-767 MR0415623

[21] M Jankins, W D Neumann, Rotation numbers of products of circle homeomorphisms, Math. Ann. 271 (1985) 381-400 MR787188

[22] K Johannson, Équivalences d'homotopie des variétés de dimension 3, C. R. Acad. Sci. Paris Sér. A-B 281 (1975) Ai, A1009-A1010 MR0391096

[23] D Kim, J Lee, Some invariants of pretzel links, Bull. Austral. Math. Soc. 75 (2007) 253-271 MR2312569

[24] T Li, R Roberts, Taut foliations in knot complements arXiv:1211.3066

[25] A V Malyutin, N Y Netsvetaev, Dehornoy order in the braid group and transformations of closed braids, Algebra i Analiz 15 (2003) 170-187 MR2052167

[26] V D Mazurov, E I Khukhro, editors, Kourovskaya tetrad: Nereshennye voprosy teorii grupp [Unsolved problems in group theory], Rossiǔskaya Akademiya Nauk Sibirskoe Otdelenie, Institut Matematiki im. S. L. Soboleva, Novosibirsk (2002) MR1956291

[27] J M Montesinos, Surgery on links and double branched covers of $S^{3}$, from: "Knots, groups, and 3-manifolds", (L P Neuwirth, editor), Ann. of Math. Studies 84, Princeton Univ. Press (1975) 227-259 MR0380802

[28] R Naimi, Foliations transverse to fibers of Seifert manifolds, Comment. Math. Helv. 69 (1994) 155-162 MR1259611

[29] Y Nakae, Taut foliations of torus knot complements, J. Math. Sci. Univ. Tokyo 14 (2007) 31-67 MR2320384

[30] P Ozsváth, Z Szabó, Holomorphic disks and genus bounds, Geom. Topol. 8 (2004) 311-334 MR2023281

[31] S P Plotnick, Finite group actions and nonseparating 2-spheres, Proc. Amer. Math. Soc. 90 (1984) 430-432 MR728363

[32] G P Scott, Compact submanifolds of 3-manifolds, J. London Math. Soc. 7 (1973) 246-250 MR0326737

[33] W P Thurston, The geometry and topology of 3-manifolds, lecture notes, Princeton University (1978-1981) Available at http://library.msri.org/books/gt3m

[34] A A Vinogradov, On the free product of ordered groups, Mat. Sbornik N.S. 25(67) (1949) 163-168 MR0031482

[35] F Waldhausen, Eine Klasse von 3-dimensionalen Mannigfaltigkeiten. I, II, Invent. Math. 3, 308-333; ibid. 4 (1967) 87-117 MR0235576

[36] C Weber, Sur une formule de R. H. Fox concernant l'homologie des revêtements cycliques, Enseign. Math. 25 (1979) 261-272 MR570312 
CIRGET, Université du Québec à Montréal

Case postale 8888, Succursale centre-ville, Montréal QC H3C 3P8, Canada

Department of Mathematics, University of Texas at Austin

1 University Station, Austin, TX 78712, USA

Department of Mathematics, UCLA

520 Portola Plaza, Los Angeles, CA 90095, USA

aclay@cirget.ca, tlid@math.utexas.edu, lwatson@math.ucla.edu

http://thales.math.uqam.ca/ aclay/, http://www.math.ucla.edu/ lwatson/

Received: 11 July 2011 Revised: 25 February 2013 